\title{
Editorial to selected papers from the International Excellence PhD School 'I. Gorini'
}

\author{
Pasquale Arpaia ${ }^{1}$, Umberto Cesaro ${ }^{1}$, Francesco Lamonaca² \\ ${ }^{1}$ University of Naples Federico II, Dept. of Information and Electrical Engineering, via Cluadio 21, Naples, 80125 (NA), Italy \\ 2 University of Calabria, Dept. of Computer Science, Modelling, Electronic and System, via P.Bucci 41C, Arcavacata di Rende, 87036 (CS), \\ Italy
}

Section: EDITORIAL

Citation: Pasquale Arpaia, Umberto Cesaro, Francesco Lamonaca, Editorial to selected papers from the International Excellence PhD School 'I. Gorini', Acta IMEKO, vol. 10, no. 4, article 3, December 2021, identifier: IMEKO-ACTA-10 (2021)-04-03

Received December 14, 2021; In final form December 14, 2021; Published December 2021

Copyright: This is an open-access article distributed under the terms of the Creative Commons Attribution 3.0 License, which permits unrestricted use, distribution, and reproduction in any medium, provided the original author and source are credited.

Corresponding author: Umberto Cesaro, e-mail: ucesaro@unina.it

\section{Dear Readers,}

This Acta IMEKO Special Issue collects the best works presented by young researchers attending the International Ph.D. School "Italo Gorini 2021", held in Naples in the period 6-10 September 2021.

The International Ph.D. School "Italo Gorini 2021" is the doctoral School promoted by the Italian associations "Electrical and Electronic Measurements Group" (GMEE) and "Mechanical and Thermal Measurements Group" (GMMT).

The School activity deals with a wide variety of issues related to measurement. The School is aimed at $\mathrm{PhD}$ students, as well as young people from research and industry fields.

The School addresses both methodological issues, in the field of science and technology related to the measures and instrumentation, and advanced problems of practical interest. Then, special attention is paid also to the impact of measures on the scientific and engineering context, which is strongly influenced by the evolution of technology in different sectors.

This year the "Italo Gorini" School has received the patronage of IMEKO and we are honoured to promote IMEKO among young brilliant scientists who will be the future of measurement science.

The works presented in this Special Issue are the extended version of those that won the "Best presentation", "Best Scientific Contribute" and "Best Application" awards during the School.

Simone Mari et al., in the paper entitled 'Measurements for nonintrusive load monitoring through machine learning approaches', propose several possible approaches for the non-intrusive load monitoring systems operating in real time, analysing them from the measurement point of view. The investigated measurement and post-processing techniques are illustrated and the results discussed.
Emanuele Buchicchio et al., in the paper 'Gesture recognition of sign language alphabet with a convolutional neural network using a magnetic positioning system' introduce a system that combines sensor-based gesture acquisition and deep learning techniques for gesture recognition providing a $100 \%$ classification accuracy. The social impact of the proposal is wide, since gesture recognition is a fundamental step to enable efficient communication for the deaf through the automated translation of sign language.

Mattia Alessandro Ragolia et al., in the paper 'A virtual platform for real-time performance analysis of electromagnetic tracking systems for surgical navigation' propose a virtual platform for assessing the performance of Electromagnetic Tracking Systems (EMTSs) for surgical navigation, showing in real time the effects of the various sources affecting the distance estimation accuracy. The implemented measurement platform provides a useful tool for supporting engineers during design and prototyping of EMTSs. A particular effort was dedicated to the development of an efficient and robust algorithm, to obtain an accurate estimation of the instrument position for distances from the magnetic field generator beyond $0.5 \mathrm{~m}$. Indeed, the main goal of the paper is to improve the limited range of current commercial systems, which strongly affects the freedom of movement of the medical team.

The paper by Leila Es Sebar et al. 'A metrological approach for multispectral photogrammetry' presents the design and development of a three-dimensional reference object for the metrological quality assessment of photogrammetry-based techniques. Such techniques are typically used in the cultural heritage field. The reference object was a $3 \mathrm{D}$ printed specimen, with a nominal manufacturing uncertainty in the order of $0.01 \mathrm{~mm}$. The object has been realized as a dodecahedron, and in each face, a different pictorial preparation has been inserted. The preparations include several pigments, binders, and varnishes, to be representative of the materials and techniques used historically by artists.

Pasquale Arpaia, Umberto Cesaro, Guest Editors

Francesco Lamonaca, Editor-in-chief 